\title{
A Problem for Goldman on Rationality
}

\author{
Francis Jeffry Pelletier \\ Department of Philosophy \\ University of Alberta
}

The central concern of Knowledge in a Social World is to restore the notion of Truth to the rightful place of glory that it had before the onslaught of those pragmatic, cultural-studying, social constructing, critical legalistic and feministic postmodernists (PoMo's, for short). As G sees it, these PoMo's have never put forward any "real" arguments for their veriphobia; and, well, how could they, since their position is committed to the "denial of Truth" and hence committed to denying that there is any such thing as a valid or a sound argument. Instead, according to G, they have artfully inculcated various types of considerations so that a defender of Truth now must swim against the entire flow of academe to even stand a chance of making his or her voice heard.

G finds six overlapping currents in this wash of anti-veritas ideas, listing them in his $\S 1.3$ and devoting a further section to each of them in turn $(\S 1.4-\$ 1.9)$. These currents spring up in various later parts of the book for further discussion, but even in these places $\mathrm{G}$ refers the reader back to the material in Chapter 1 as refuting them. (Insofar as a current that is not based on any consideration of Truth, validity, or soundness is susceptible of refutation.) I think it is fair to say that G does not really intend that his comments about the anti-veritas ideas will be taken as definitive by the veriphobes, but rather that he believes the strength of his positive account in the rest of the book (which accords Truth a central role) will sway all neutral readers into a belief of the usefulness of Truth in an account of social knowledge. Still, I think many readers...even veriphiles...will find the considerations that $\mathrm{G}$ brings against the six currents to be disappointingly short.

I want to consider some of the currents in the stream of PoMo ideas. As I said, G's six currents overlap at various points, and each of the currents has sub-flows. This means that we might put 
together sub-flows from different currents in such a way as to find a somewhat different current in the PoMo stream than was found by G. To lay my cards on the table at the outset: I am a veriphile, I am an anti-PoMo, I am a believer in individual epistemology and hold that social epistemology is entirely reducible without remainder to the knowledge of individuals. Except possibly for this last point I am in agreement with G. My task in this short commentary should be seen as trying to bolster one small part of his overall position by making one PoMo consideration as strong as possible and asking $\mathrm{G}$ to show that it still fails. Of course, no answer of G's will convince a PoMo because they do not think that considerations of truth, validity, consistency, and the like are relevant to what should be believed. But perhaps it will help with a few of G's neutral readers.

\section{The Argument from Bias:}

PoMo's in general, at least in the version given by G, deny the reality of rationality. The various currents that $\mathrm{G}$ identifies all have the goal of relegating the desire for Truth and the desire for the search for Truth into the backwaters of human endeavor. The underlying message one gathers from G's presentation is that PoMo's not only deny that there is any such thing as Truth but additionally they attribute any belief in it either to ignorance or to sinister motives. The sixth of the PoMo currents detected by $\mathrm{G}$ is that

Truth cannot be attained because all putatively truth-oriented practices are corrupted and biased by politics or self-serving interests.

G's rebuttal of this current in PoMo thought is simple: he claims that it depends on two very implausible assumptions, namely (a) that all belief is driven by motivational biases, and (b) that these biases are in conflict with the Truth. G claims that (a) is wrong, in general, because one does not have the voluntary choice to believe or disbelieve something and hence it cannot be due to 
motivational biases. He claims that (b) is wrong because it is simply not true that knowing the truth runs generally against one's interests (although speaking the truth might run against one's interests).

In $§ 1.9$, where this argument is discussed, there is very little more said about either it or about these two assumptions, except for a discussion of the role of case studies in the history of science. His analysis of the issue in this section of the book is that all the plausibility of The Argument from Bias comes from its application to political discussion, where a conflict might be seen between one's interests and speaking the truth. But, G continues, such a generalization is not correct for knowledge and truth in general. There are, of course, researchers who think that case studies in the history of science show that "any theory can always be retained as long as its defenders have enough institutional power to explain away potential threats to it”. In his discussion of these case studies, G acknowledges that politics and interests have played a crucial role in the development and acceptance of scientific ideas-something no one has ever questioned-but he denies that this is at all relevant to the issue of Truth. To be relevant, he says, would require that politics and interests were causally efficacious for the scientific beliefs and not merely temporally coincident with them. And this would require establishing the counterfactual thesis that the beliefs would not have occurred had the politics or interests of the situation been different. G denies that the case studies have ever established this. And anyway, even if it could be established, this wouldn't be enough to show that the acceptance of these ideas-especially over a long period of time-stemmed from these same motivations.

This last item, that long-term acceptance is independent of motivational bias, is a theme that I believe G should have emphasized more. My view of long-term acceptance is like the following, and although I believe this is in accord with G's general position, I wish he had more explicitly addressed the issue. Imagine a flat plane upon which various theses about the world are inscribed. 
Imagine further that the choice of which of these theses are accepted by an individual (or group of people) is indicated by the position of a marble (or all of the group's individual marbles) on this plane. Certain factors deform the plane: sometimes a heavy weight is placed at a location, making that point lower and the marbles more likely to roll to that place; or maybe the plane is forced upwards at some location and the marbles tend to roll away from that location. A person's (or group's) beliefs over time are rather like rolling the marble from one point on the plane to see where it will eventually end up...perhaps with the occasional shake of the plane to represent violent new starts. My view is that individual's motivational biases are best represented as weights ("attractors") on this plane that make the marbles tend to roll towards them. And there perhaps are other motivational features that function as "repellors". The individual's marble will roll towards the attractors and away from the repellors, as will the group of marbles representing the group or social beliefs. But as I see it, the greatest attractor is Truth; and after a sufficiently long time all (or close to all) the marbles will end up in the deformity caused by the great weight of Truth. The marbles will have escaped from all the minor attractors and repellors, and will have been drawn toward the Truth.

Of course, the world of beliefs is not really a plane with various theses inscribed at different locations, and biases and the Truth are not really heavy weights placed on the plane to deform it into the third dimension. No: the world of beliefs is really a high-dimensional hyperplane and biases and the Truth are really attractors/repellors that deform the hyperplane in a higher dimension. However, our beliefs, especially our beliefs over time, are just marbles that roll around in this hyperplane and are attracted/repelled by these various forces. But again, the factor that accounts for the majority of the effect is the super-attractor, Truth. 


\section{Gigerenzer/Cohen/Cosmides}

G continues his discussion of the PoMo current which uses The Argument from Bias in his Chapter 8 , and devotes $\S 8.3$ to a further rebuttal. In this Section, $\mathrm{G}$ concentrates on specific biases that might be mentioned by PoMo's (although I think they never have been) but which have been the subject of a dispute in Cognitive Science, concerning the nature of rationality and what it means to be rational.

Biases of the sort that affect one's acceptance or rejection of "factually-oriented" statements come in two flavors: hot and cold. The hot biases are the ones caused by emotions, desires, special interests that "blind" one to "the facts", and the like. Such a bias will make one believe that one's spouse is faithful, despite all the clear evidence to the contrary; they will make one "refuse to even consider" the benefits of gun registration, and the like. But it is not these hot biases that I wish to consider here; instead, cold biases are those which are due to the inbuilt mechanisms of human cognitive functioning. Thus, even in cases where one is attempting to "dispassionately investigate" some phenomenon, one's inbuilt cold biases may lead one to "false" answers. PoMo's could use this fact (if it is a fact) to argue that the notion of Truth is inevitably bound to be dependent on these cold biases and to have no independent use or validity. So far as I know, this exploitation of cold biases has not been followed up on by PoMo's, but it is clear that it could be, possibly with even more plausibility than their employment of the similar case of hot biases. After all, with hot biases we want to say that they can be overcome by "cooling down" or "stepping back" and letting one's underlying rationality come to the fore. But cold biases offer no such method, for the biases are built into the very structure of our rational nature.

Long ago it was discovered that many (most) people are "remarkably bad" at simple logic problems and that they similarly are "distressingly unable" to perform even the most simple tasks of estimating probabilities. Although such a discovery comes as no surprise to teachers of elementary 
logic and elementary probability theory, the detailed psychological study of this "shortcoming" took off in the deductive logic realm with the publication of Wason (1966) and in the probabilistic reasoning realm with the works of Kahneman \& Tversky (there have many joint works, see Nisbett \& Ross 1980 or Evans 1987 for summaries). In 1981 Cohen challenged these findings partially on the grounds that rationality was defined to be what people's reasoning competence allows them to do. Therefore, he concluded, it cannot be true that people are "systematically biased". Members of this "optimistic camp" (optimistic because they believe that people are inherently rational) have since that time been engaged in the exercise of explaining how the original experiments are in some way wrong. $\mathrm{G}$ is a member of this optimistic camp, and he employs some of the standard “explanations" with an eye to showing that the PoMo's are wrong in their appeal to this sort of bias as a justification of denying Truth. His general conclusion is that cold biases (a) do not happen as often as PoMo's would require, nor (b) are they necessarily detrimental to the use of Truth when they occur.

Here is a cross-section of the optimistic suggestions concerning how and why optimists think the alleged biases do not really count against rationality as a route to Truth. We'll see that all of them, including the one advocated by G, are variations on the same theme; and I will indicate why I think this reply to PoMo's just can't be successful. Cohen (1981) postulated that the probabilistic reasoning that was actually demonstrated by subjects - the "bad reasoning" which Kahneman \& Tversky had uncovered - was in fact the correct way to reason. This way of reasoning was formalized as a "Baconian probability calculus" which Cohen then put forward as the correct way to reason probabilistically (as opposed to the Baysean calculus that was assumed by Kahneman \& Tversky when they made their judgment that people were inherently biased). A variant on this sort of position is taken by Gigerenzer (see for example his 1991, 1994, 1998). Gigerenzer believes that 
the flaw in the Kahneman \& Tversky experiments was that they were trying to get subjects to give the probability of single events (such as the probability that Linda is a feminist bankteller) instead of the frequency of a type of event happening within a larger space of events. In a series of very compelling experiments Gigerenzer has shown how to make (most) of the alleged biases disappear by suitably rephrasing the experiments in terms of frequency judgments instead of single-event probabilities. The difference between Cohen's and Gigerenzer's strategies is that Cohen wanted to do a wholesale replacement of Baysean probability theory, whereas Gigerenzer agrees with the predictions of Baysean theory in those cases where there is a frequency interpretation of the case. He only disagrees with a certain portion of Baysean theory, viz., the probability of single events; and his claim is (a) that in the good portion of Baysean theory (where there are no single-event probabilities) people will agree with Baysean theory if the material is presented to them in a frequency manner, (b) even in the bad portion of Baysean theory, if the problem can somehow be rephrased as a frequency problem then people will agree with the Baysean answer, and (c) all the remaining cases are those where subjects are asked to find the probability of a single event and this is simply not a well-defined notion (so it is not surprising that subjects cannot do it coherently). Like Cohen, Gigerenzer is challenging Bayseanism as being the normatively correct theory of probability.

In the realm of deductive reasoning a similar battle is being waged. Wason's (1966) results show that subjects do not follow classical logic in their assessment of simple reasoning involving conditionals and negations. So far as I am aware no one has really taken up the type of answer that Cohen gave for the probabilistic reasoning case - to construct a new deductive logic that obeys the results obtained in the experiments and claim that this is the normatively correct logic. (Although there have been some mutterings about "fuzzy logic", "relevant logic", and the like.) Instead, the 
main force in the literature has been more akin to Gigerenzer's strategy: find particular types of problems that subjects can perform well on (well, as measured by classical logic) and then claim that this is the normatively correct notion of (deductive) rationality. The types of problems that people do not perform well are then relegated to the realm of "not well-defined problems", much as the probability of single events is disparaged by Gigerenzer. In the deductive realm this course is championed most feverently by Cosmides (1989, see also Cosmides \& Tooby 1987, 1994). Her claim is that people can perform well on the Wason task so long as the logic problems ask about "social interactions", particularly about whether some "cheating" is going on. If we give people this problem:

From "No one who has not paid their dues can enter the meeting-house" and "Joe is allowed to enter the meeting-house" can we infer "Joe has paid his dues"?

then everyone will correctly answer "yes". Yet, if the question is put:

From "No non-F's are G's" and "a is a G" can we infer "a is an F"?

we find that most people will answer this incorrectly (according to classical logic). Cosmides' position is, like Gigerenzer's, that these "abstract" questions simply do not have any "rational" answer, because the notion of (deductive) logical rationality is only defined for problems of the “cheating detection" variety. (And she has an evolutionary-psychology justification for this.) The background logic of her theory agrees with classical logic in those cases where it is defined. But there are other cases where it is not defined and people's performance on these cases cannot be used to argue for or against human rationality.

According both to Gigerenzer and Cosmides, (a) there is an objective normative standard in the fields of probabilistic and deductive reasoning, and (b) people's underlying rational competence matches this standard. The (a) aspect reiterates their commitment to veritism and the (b) aspect 
demonstrates their commitment to optimism. And it is to this picture that $\mathrm{G}$ cleaves. But can it really be used as an argument against the PoMo current about biases? It seems to me to have at least the following problem. The main idea of Gigerenzer and Cosmides is to presuppose the existence of objective rational standards, and then to use human performance to show the restrictions on the scope of these standards. It is true, they say, that people do not perform logical operations in accordance with the dictates of classical logic (e.g., they misapply contraposition when problems are presented in "abstract formats") nor do the perform appropriate estimations of probabilities in accordance with Baysean theory (e.g., they misapply information about "base rates" if the information is given to them as a probability rather than a frequency). But this does not show that there are biases, they say; for the correct conclusion is to deny that human rationality extends to these cases. (Such cases are instead seen as some sort of "theoretical extension" of the basic theory of human rationality in the same way that imaginary numbers constitutes a theoretical extension to the ordinary notion of number.)

I do not see how adopting the Gigerenzer/Cosmides viewpoint can serve as an argument against PoMo. For, the main idea of Gigerenzer/Cosmides is to presuppose there to be Truth and to then use this to show the reach of human rationality, whereas the PoMos are using biases to show the non-existence of Truth. As an analogy, consider arguing with someone who claims that everyone believes in a supreme being. You proceed by pointing to those who have denied believing and have said so in their writings; and you might even point to yourself. But just when you think the opponent is bound to give in (after all... who better than you to know whether you believe in a supreme being?), s/he takes the "whatever you hold to be most important-that's what you hold to be a supreme being" move. And just as we find this an intellectually unsatisfying maneuver, so too should PoMo's find unsatisfying G's definition (or the Gigerenzer/Cosmides definition) of 
'rationality' as whatever aspect of performance agrees with some pre-given normative standards. After all, PoMo's have not yet even admitted there to be any such normative standards. I would like to see $\mathrm{G}$ address the "pure issue" of the possibility of (cold) biases making it impossible ever to know whether Truth has been attained without presupposing the existence of Truth. G recognizes, $\mathrm{p}$. 234, that he has "not refuted the heuristics-and-biases approach root and branch." But I would say that even if he could refute the Wason/Kahneman/Tversky tradition, his methodology presumes that there is a normative standard of Truth, and therefore this refutation would not (should not!) dissuade the PoMo's.

\section{The Denial of Epistemic Privilege}

Another current $\mathrm{G}$ finds in the PoMo river is the denial of epistemic privilege:

There are no privileged epistemic positions, and no certain foundations for beliefs. All claims are judged by conventions or language games, which have no deeper grounding. There are no neutral, transcultural standards for settling disagreements.

Having introduced my main worry with this aspect of G's work, let me carry it a bit further into this other current of PoMo thought. In $§ 1.7$, where this current is considered, G spends much of his time discussing Rorty's (1979) arguments against “privileged representations”, rightly remarking that even if Rorty's claims here were right they would not tell against any of the other versions of epistemology on the market, especially G's own "reliabilism". A second sub-flow of this current concerns the idea that claims are always judged by conventions, perhaps the conventions that are part of the "language game" being played by the agents or by the agreement of others in the culture. It would follow from this that there can be no independently-justified cross-cultural standards. Once again $\mathrm{G}$ is swift in his dismissal of the claim (p. 29) 
Careful reflection on judgments of justification suggests-as reliabilism maintains-that a belief is considered justified if it is arrived at by processes or practices that the speaker (or the community) regards as truth conducive. ...Thus, judgments of justification are not without grounding, nor are they purely conventional. They are grounded precisely on appeals to truth conduciveness. Furthermore, there seems to be nothing arbitrary in a concept of justification tied to truth conduciveness.

The reason there is "nothing arbitrary" in this is that "people have an interest in truth" and those beliefs "formed by truth-conducive processes are more likely to be true." A final sub-flow of this current concerns the notion of "agreement". G thinks that PoMo's believe that veriphiles require agreement for their notion of rational justification. G also thinks that PoMo's believe such agreement to be impossible because they also believe there to be no transcultural principles for settling disagreements among different communities. G tries to calm this sub-flow by distinguishing the "ultimate aim" of epistemic practices from the specific methods adopted in pursuit of these aims. He claims that it has always been the pursuit of Truth that different cultures have aimed at, although these different cultures adopt many, many different and radically opposed methods in their pursuit of Truth.

Here's a problem for this picture. I might also mention that it is a problem for my view that I expressed as the simile of marbles rolling on a plane. Both G's view and the one I mentioned require that Truth be a causal factor. We have just seen that G makes Truth be the cause of a pursuit by people of any culture at all; and in my simile, Truth played the role of deforming the plane so that the marbles representing people's beliefs would ultimately roll toward that place on the plane. In my picture I also had the biases and motivations be deforming features of the plane. Now, it's pretty clear how biases and other motivations can be causally efficacious in producing belief. But the same cannot be said about Truth. It is, I think we would all agree, a non-natural property. It certainly is 
not a property that can easily be thought as exerting some mysterious force that attracts people's mental states. Indeed, it seems to be qualitatively or categorially the wrong sort of property to do this. It is one thing to say that the cause for $82 \%$ of the people asked thinking that Linda is more likely to be a feminist bankteller than to be a bankteller is due to some mental short-circuit or bias to ignore conjunctions (or whatever). But what will then be the cause for the other 18\%? Don't we need some explanation on the same "level" as bias to explain their performance? How can we just simply appeal to the truth of the matter as the operative causal factor? (This is an adaptation of the "strong programme" requirement of symmetry of explanation-see Barnes \& Bloor 1982 for further explanation. Although G mentions Barnes, Bloor, and Barnes \& Bloor, he does not discuss this aspect of the strong programme.)

I would be very grateful to $\mathrm{G}$ if he could give a convincing answer to this problem. 


\section{References}

Barnes, B. \& D. Bloor (1982) "Relativism, Rationalism, and the Sociology of Knowledge” in M. Hollis \& S. Lukes (eds.) Rationality and Relativism (Cambridge: MIT Press) pp. 21-47.

Cohen, L.J. (1981) "Can Human Irrationality be Experimentally Demonstrated?" Behavioral and Brain Sciences 4: 317-370.

Cosmides, L. (1989) “The Logic of Selection: Has Natural Selection Shaped how Humans Reason? Studies with the Wason Selection Task" Cognition 31: 187-276.

Cosmides, L. \& J. Tooby (1987) "From Evolution to Behavior: Evolutionary Psychology as the Missing Link" in J-P. Dupré (ed.) The Latest on the Best: Essays on Evolution and Optimality (Cambridge: MIT Press) pp. 277-306.

Evans, J. (1987) Bias in Human Reasoning: Causes and Consequences. (Hillsdale, NJ: Lawrence Erlbaum).

Gigerenzer, G. (1991) “How to make Cognitive Illusions Disappear: Beyond 'Heuristics and Biases"” European Review of Social Psychology 2: 83-115.

Gigerenzer, G. (1994) "Why the Distinction between Single-event Probabilities and Frequencies is Important for Psychology (and Vice Versa)" in G. Wright and P. Ayton (eds.) Subjective Probability (NY: John Wiley \& Sons), pp. 129-161.

Gigerenzer, G. (1998) “Ecological Intelligence: An Adaptation for Frequencies” in D. Cummins \& C. Allen (eds.) The Evolution of Mind (NY: Oxford University Press), pp. 9-29.

Kahneman, D., \& A. Tversky (1973) “On the Psychology of Prediction” Psychological Review 80: 237-251.

Kahneman, D., P. Slovic, \& A. Tversky (1982) Judgment Under Uncertainty: Heuristics and Biases. (Cambridge: Cambridge UP). 
Nisbett, R. \& L. Ross (1980) Human Inference (Englewood Cliffs: Prentice-Hall).

Rorty, R. (1979) Philosophy and the Mirror of Nature (Princeton: Princeton Univ. Press).

Wason, P. (1966) "Reasoning” in B. Foss (ed.) New Horizons in Psychology London: Penguin Books). 